\title{
Targeted resequencing reveals rare variants enrichment in multiple
}

\section{sclerosis susceptibility genes}

Elia Gil-Varea ${ }^{1}$, Nino Spataro ${ }^{2}$, Luisa María Villar ${ }^{3}$, Amalia Tejeda-Velarde $^{3}$, Luciana Midaglia $^{1}$, Fuencisla Matesanz ${ }^{4}$, Sunny Malhotra $^{1}$, Herena Eixarch ${ }^{1}$, Nikolaos Patsopoulos $^{5}$, Óscar Fernández ${ }^{6}$, Begoña Oliver-Martos ${ }^{6}$, Albert Saiz ${ }^{7}$, Sara Llufriu ${ }^{7}$, Lluís Ramió-Torrentà ${ }^{8}$, Ester Quintana ${ }^{8}$, Guillermo Izquierdo ${ }^{9}$, Antonio Alcina ${ }^{4}$, Elena Bosch $^{2,10}$, Arcadi Navarro ${ }^{2,11,12}$, Xavier Montalban ${ }^{1}$, Manuel Comabella ${ }^{1 *}$

Author Affiliations: ${ }^{1}$ Servei de Neurologia-Neuroimmunologia, Center d'Esclerosi Múltiple de Catalunya (Cemcat). Institut de Recerca Vall d'Hebron (VHIR). Hospital Universitari Vall d'Hebron. Universitat Autònoma de Barcelona. Barcelona, Spain. ${ }^{2}$ Genetics Laboratory, UDIAT-Centre Diagnòstic. Parc Taulí Hospital Universitari. Institut d’Investigació i Innovació Parc Taulí I3PT. Universitat Autònoma de Barcelona. Sabadell, Spain. ${ }^{3}$ Departments of Immunology and Neurology, Multiple Sclerosis Unit, Hospital Ramon y Cajal, (IRYCIS), Madrid, Spain. ${ }^{4}$ Department of Cell Biology and Immunology, Instituto de Parasitología y Biomedicina "López Neyra", Consejo Superior de Investigaciones Científicas (IPBLN-CSIC), Granada, Spain. ${ }^{5}$ Department of Neurology, Brigham \& Women's Hospital and Harvard Medical School, Boston, Massachusetts. ${ }^{6}$ Neuroimmunology and Neuroinflammation Group. Instituto de Investigación Biomédica de Málaga-IBIMA. UGC Neurociencias. Hospital Regional Universitario de Málaga, Spain. ${ }^{7}$ Servicio de Neurología, Hospital Clinic and Institut d'Investigació Biomèdica Pi i Sunyer (IDIBAPS), Barcelona, Spain. ${ }^{8}$ Neurodegeneration and Neuroinflammation Group, Girona Biomedical Research Institute (IdIBGi). Department of Medical Sciences, Faculty of Medicine, University of Girona, Spain. ${ }^{9}$ Departamento de Neurología, Hospital Universitario Virgen Macarena, Sevilla, Spain. 
${ }^{10}$ Centro de Investigación Biomédica en Red de Salud Mental (CIBERSAM), Reus, Spain. ${ }^{11}$ Centre de Regulació Genòmica (CRG). Barcelona, España. ${ }^{12}$ Institució Catalana de Recerca i Estudis Avançats (ICREA), Cataluña, Spain.

*Corresponding author: Manuel Comabella; Center d'Esclerosi Múltiple de Catalunya (Cemcat). Institut de Recerca Vall d'Hebron (VHIR). Hospital Universitari Vall d'Hebron. Pg. Vall d'Hebron 119-129 08035 Barcelona, Spain. Phone: +34932746834, Fax:+34932746084. e-mail: manuel.comabella@vhir.org

\section{Grant numbers (funding)}

This work was supported by grants from the Fondo de Investigación Sanitaria (FIS; grant number PI12/02229; Ministry of Science and Innovation, Spain), Agencia Estatal de Investigación (AEI) and Fondo Europeo de Desarollo Regional (FEDER; grant number BFU2016-77961-P), Direcció General de Recerca - Generalitat de Catalunya (2017-SGR00702), Unidad de Excelencia María de Maeztu (MINECO; MDM-2014-0370), and Vall d'Hebrón Institut de Recerca (predoctoral grants programme 2014). 


\section{ABSTRACT}

Although genome-wide association studies have identified a number of common variants associated with multiple sclerosis (MS) susceptibility, little is known about the relevance of rare variants. Here, we aimed to explore the role of rare variants in fourteen MS risk genes (FCRL1, RGS1, TIMMDC1, HHEX, CXCR5, LTBR, TSFM, GALC, TRAF3, STAT3, TNFSF14, IFI30, CD40 and CYP24A1) by targeted resequencing in an Iberian population of 524 MS cases and 546 healthy controls. Four rare variants-enriched regions within CYP24A1, FCRL1, RGS1 and TRAF3 were identified as significantly associated with MS. Functional studies revealed significantly decreased $R G S 1$ gene expression levels in peripheral blood mononuclear cells (PBMC) from MS patients with RGS1 rare variants compared to non-carriers, whereas no significant differences in gene expression were observed for CYP24A1, FCRL1 and TRAF3 between rare variants carriers and noncarriers. Immunophenotyping showed significant decrease in RGS1 expression in peripheral blood B lymphocytes from MS patients with $R G S 1$ rare variants relative to non-carriers. Lastly, PBMC from MS patients carrying RGS1 rare variants showed significantly lower induction of $R G S 1$ gene expression by interferon-beta compared to MS patients lacking $R G S 1$ variants. The presence of rare variants in $R G S 1$ reinforce the ideas of high genetic heterogeneity and a role of rare variants in MS pathogenesis.

Keywords: multiple sclerosis, targeted DNA sequencing, single nucleotide polymorphisms, rare variants, $R G S 1$, interferon- $\beta$ 


\section{INTRODUCTION}

Multiple sclerosis (MS [MIM: 126200]) is a heterogeneous inflammatory disease of the central nervous system characterized by autoimmune events, myelin loss and neurodegenerative outcomes (Ebers, 2008). Although the etiology of MS remains poorly understood, several lines of evidence suggest that the interaction between genetic and environmental factors has a great impact in disease pathogenesis (Ascherio, 2013). Thereby, because of environmental exposures, genetically susceptible individuals may develop MS (Kakalacheva \& Lünemann, 2011). This genetic susceptibility is not based upon a single gene, but upon the cumulative effect of a large number of allelic variants, making MS as a disease of highly polygenic inheritance (Oksenberg, 2013). Over the last years, genome-wide association studies (GWAS) have significantly contributed to shade light on the genetic component of MS and identified a large number of common variants associated with MS risk, as well as their potential target genes (Beecham et al., 2013; Patsopoulos et al., 2019; Sawcer et al., 2011). To be more precise, by means of international collaborative efforts involving large cases and controls cohorts, more than 200 common genetic variants outside the major histocompatibility complex (MHC) have been identified to be associated with MS risk to date (Patsopoulos et al., 2017). However, GWAS also show that only a limited proportion of MS heritability can be explained by the common variants usually tagged by single nucleotide variant arrays, which has led to the so-called missing heritability concern (Manolio et al., 2009). This concern could be partially addressed taking into consideration not only common susceptibility variants with small effect sizes, but also rare variants that, while probably having larger effects, have such low frequencies that it has not been possible to link them to disease susceptibility using GWAS (Lettre, 2014; Saint Pierre \& Genin, 2014). Although recently a large-scale study has unveiled the association of a number of individual low-frequency coding 
variants with MS risk (Mitrovič et al., 2018), to our knowledge no studies so far focus on whether an enrichment of rare variants in cases versus controls could be functionally relevant and thus contribute to MS susceptibility. Bearing this in mind, in the present study we aimed to investigate the role of rare variants in MS by resequencing a selected set of MS susceptibility genes, analysing rare variants enrichment in them and exploring their potential functional consequences in the pathogenesis of the disease. 


\section{MATERIALS AND METHODS}

\section{Resequencing cohort}

A total of 1,095 individuals (545 patients with relapse-onset MS and 550 healthy controls) participated in the study. The 545 MS patients were recruited from 5 Spanish MS centers [Málaga $(n=75)$; Barcelona - Cemcat $(n=358)$; Granada $(n=14)$; Barcelona - Hospital Clinic ( $\mathrm{n}=52)$; Girona ( $\mathrm{n}=46)]$. All MS patients fulfilled the 2010 McDonald criteria (Thompson et al., 2018). The 550 healthy controls were recruited from the following MS centers [Málaga $(n=79)$; Barcelona - Cemcat $(n=411)$; Granada $(n=14)$, Girona $(n=46)]$.

\section{Candidate genes selection}

Fourteen candidate genes previously associated with MS by GWAS (Beecham et al., 2013; Patsopoulos et al., 2019; Sawcer et al., 2011) were selected based on the accomplishment of one or two of the following criteria: (i) their differential gene expression between MS patients and healthy controls in microarray studies performed by our group in peripheral blood mononuclear cells (PBMC) (unpublished data; criterion 1; see Supplementary Text); (ii) their potential contribution to the pathogenesis of MS according to the literature beyond its association with MS risk in the GWAS study (criterion 2). The 14 genes selected for DNA resequencing were the following: Fc receptor like 1 (FCRL1; criterion 2: Comabella et al., 2016), regulator of G protein signaling 1 (RGS1; criterion 1: up-regulated in MS patients versus controls; criterion 2: Tran et al., 2010), translocase of inner mitochondrial membrane domain containing 1 (TIMMDC1; criterion 1: down-regulated in MS patients versus controls), hematopoietically expressed homeobox (HHEX; criterion 1: down-regulated in MS patients versus controls), C-X-C motif chemokine receptor 5 (CXCR5; criterion 2: Klimatcheva et al., 2015), lymphotoxin beta receptor (LTBR; criterion 2: Inoue et al., 
2016), Ts translation elongation factor, mitochondrial (TSFM; criterion 2: Alcina et al., 2013), galactosylceramidase (GALC; criterion 2: Scott-Hewitt et al., 2017), TNF receptor associated factor 3 (TRAF3; criterion 1: up-regulated in MS patients versus controls), signal transducer and activator of transcription 3 (STAT3; criterion 2: Benveniste et al., 2014), TNF superfamily member 14 (TNFSF14; criterion 1: down-regulated in MS patients versus controls; criterion 2: Malmeström et al., 2013), IFI30, lysosomal thiol reductase (IFI30; criterion 2: West and Cresswell, 2013), CD40 molecule (CD40; criterion 2: Chen et al., 2016) and cytochrome P450 family 24 subfamily A member 1 (CYP24A1; criterion 2: Ramasamy et al., 2014).

\section{Capture, resequencing and variant calling}

Genomic DNA from peripheral blood samples was obtained using standard methods and $5 \mu \mathrm{g}$ of DNA per subject were used to construct the Illumina HiSeq DNA libraries as follows.

For each selected gene, coordinates for each possible exon were obtained from BioMart (Ensembl genes 67, GRCh37.p7) and then extended in order to include putative splicing sites (with 100 intronic base pairs at both ends of each exon) and any possible regulatory sequence (retrieved from Ensembl) overlapping them. We also targeted $2000 \mathrm{bp}$ upstream from the transcription start site of each gene to capture the promoter region, for a total of 140,144 target bases submitted to design. All target capturing and sequencing procedures were performed at BGI Hong Kong (now BGI Tech Solutions). Briefly, genomic DNA was randomly fragmented with a Covaris instrument into fragments with a base pair peak of 150 to $200 \mathrm{bp}$. Following NimbleGen's recommendations for capturing Illumina DNA libraries, adapters were ligated to both ends of the resulting fragments. Extracted DNA 
was then amplified by ligation-mediated PCR (LM-PCR), purified, and hybridized to the NimbleGen human custom array for enrichment; non-hybridized fragments were then washed out. Both non-captured and captured LM-PCR products were subjected to quantitative PCR to estimate the magnitude of enrichment. Each captured library was then loaded on a Hiseq2000 platform, and high-throughput sequencing for each captured library with paired-end reads of $90 \mathrm{bp}$ was performed independently to ensure that each sample met the desired average fold-coverage.

Raw reads were first mapped to the human reference genome (hg19) using the BWA aligner and subsequently processed using the GATK pipeline in order to realign reads around indels, remove PCR duplicates and perform base quality score recalibration. The mean coverage per sample and target was $85.64 \times$ and only 3 of the initial 141 fragments to target yielded a mean coverage $<5 \times$ over different samples and were removed from our analysis. Variant discovery was performed using the Haplotype Caller tool of GATK with the parameters for variants and indel filtering described on GATK documentation for target sequencing experiments. We identified a total of 2,082 biallelic polymorphic variants which included 179 indels and 1,903 variants. The transition/transversion ratio in our final dataset was 2.2. For variant annotation, we used ANNOVAR (Wang, Li, \& Hakonarson, 2010), a tool suited for functional annotations of variants detected from high-throughput sequencing data. Categorical functional base annotations were obtained from 12 pathogenicity predictors through ANNOVAR. In particular, SIFT, Provean, MutationAssessor, MutationTaster, LRT, FATHMM, MCAP, fathmmMKL_coding, MetaLR and MetaSVM variant classifications were retrieved from the dbnsfp database (version 35c), whereas those of PolyPhenHDIV and PolyPhenHVAR were obtained from the ljb database (version 26) as available at ANNOVAR. Moreover, we also used spliceAI (Jaganathan et al. 2019; https://github.com/Illumina /SpliceAI) to annotate splice 
variants. To consider different potential functional impact levels, we distinguished between the following (partly overlapping) categories: coding, code-altering, putative code-damaging, regulatory, promoter and UTR variants. Coding, regulatory, promoter and UTR variants comprise all variants detected on exons, regulatory regions, promoters and UTRs as downloaded from Ensembl and used for the design, respectively. We classified as "code-altering variants" all non-synonymous, frameshift, nonsense and splicing variants identified in the coding regions. Finally, we considered as "putative code-damaging variants" all nonsense, frameshift and splicing variants together with all the non-synonymous variants predicted to have pathological consequences by at least either 2, 6 or 12 pathogenicity prediction algorithms as implemented in ANNOVAR (see Supplementary Table 1).

\section{Principal component analysis (PCA) and quality control}

Population substructure within cases and controls was investigated by means of principal component analysis (PCA). PCA was performed using SmartPCA software package and outputting the first 10 principal components (-k10). Only 1 sample was found as an outlier over the first component and was removed from further analysis, while 4 samples were found as outliers over the $5^{\text {th }}$ component and removed from further analysis. To test the presence of related individuals, we counted the number of genotype differences at all the called variant between each possible pair of samples. A total of 42 sample pairs were showing a number of differences lower than 55 and for these pairs we discarded the sample showing the lowest coverage. In total, 1,070 samples were retained for further analysis, comprising 524 MS cases (214 males and 310 females) and 546 controls (230 males and 316 females). A summary of demographic and clinical characteristics of the final resequencing cohort is shown in Supplementary Table 2. 


\section{Rare variants association analysis}

To assess the contribution of rare variants to MS, a number of association tests for enrichment with rare variants were performed using the Variant Association Tools options within the Variant Tools software version 2.0 (http://varianttools.sourceforge.net/). The tests were Combined and Multivariate Collapsing (CMC), $c(\alpha)$, Kernel Based Adaptive Clustering (KBAC), Replication Based Test (RBT), RareCover, Variable Thresholds method (VT), Weighted Sum Statistic (WSS) and data-adaptive Sum test (aSUM). For these analyses, we considered as rare variants those with $\mathrm{MAF} \leq 0.5 \%$ in the whole dataset of 1,070 used individuals. Collapsing methods were performed considering all the genes under analysis jointly, but also for each single gene separately and on the different groups of variants described above. The direction of the association was inferred computing the average number of rare variants in cases and controls.

\section{Determination of gene expression levels in PBMC}

mRNA expression levels for $R G S 1, T R A F 3, C Y P 24 A 1$ and FCRL1 were determined in PBMC from a subgroup of untreated MS patients of the resequenced cohort carrying either one or more rare variants or any rare variant in the reported rare variants-enriched region for each corresponding gene, and compared with the expression levels observed in rare variants non-carriers. Expression experiments were performed only for those genes with at least two collapsing tests significant at 0.05 level. Briefly, PBMC were isolated by Ficoll-Isopaque density gradient centrifugation (Gibco BRL, Life Technologies LTD, Paisley, UK) and stored in liquid nitrogen until used. Total RNA was extracted from PBMC using TRIzol (GIBCO-BRL, Life Technologies, Grand Island, NY) and cDNA synthesized using the High-Capacity cDNA Reverse Transcription Kit (Applied 
Biosystems, Foster City, CA). RGS1, TRAF3, CYP24A1 and FCRL1 expression levels were determined by real-time PCR using TaqMan ${ }^{\circledR}$ probes specific for each gene (Hs01023772_m1, Hs00936781_m1, Hs00167999_m1 and Hs00364705_m1, respectively; Applied Biosystems). The housekeeping gene glyceraldehyde-3-phosphate dehydrogenase (GAPDH) was used as an endogenous control (Applied Biosystems). Assays were run on the ABI PRISM $® 7900 H T$ system (Applied Biosystems) and data were analysed with the $2^{-\Delta \Delta C T}$ method (Livak \& Schmittgen, 2001). Results were expressed as fold-change in gene expression in MS patients with rare variants relative to those without rare variants in the corresponding gene.

\section{RGS1 immunophenotyping in PBMC subpopulations}

A total of 11 untreated MS patients classified according to the presence $(n=4)$ or absence $(\mathrm{n}=7)$ of $R G S 1$ rare variants were selected from the resequencing cohort. PBMC were isolated and stored as described above. A total of $1 \times 10^{6}$ cells were stained with different combinations of antibodies to analyze the expression of RGS1 in selected PBMC subpopulations by flow cytometry. First, cells were surface stained using the following antibodies (from BD Biosciences, San Diego, CA, USA): anti-human CD45-V500, antihuman CD3-PerCP, anti-human CD8-APC·H7, anti-human CD14-FITC, anti-human CD19-PE·Cy7, anti-human CD56-APC, anti-human CD25-APC, and anti-human CD127-BV421. Cells were then fixed/permeabilized with Cytofix/Cytoperm ${ }^{\mathrm{TM}}$ (BD Biosciences) according to the manufacturer's protocol and RGS1 intracellular staining was performed using a rabbit polyclonal anti-RGS1 antibody (Novus Biologicals, Littleton, CO) and then with PE-conjugated donkey anti-rabbit IgG secondary antibody (Biolegend). PBMC were acquired with the BD FACSCanto ${ }^{\mathrm{TM}}$ II flow cytometer (BD Biosciences), and the FACSDiva software V.8.0 (BD Biosciences) was used for analysis. 
Cells were gated by combining FSC and SSC parameters. Monocytes were then gated as $\mathrm{CD} 45^{+} \mathrm{CD} 14^{+} \mathrm{CD} 3^{-}$, B lymphocytes as $\mathrm{CD} 45^{+} \mathrm{CD} 3^{-} \mathrm{CD} 19^{+}$, NK cells as $\mathrm{CD} 45^{+} \mathrm{CD} 3^{-}$ $\mathrm{CD}^{+} 6^{+}, \mathrm{NKT}$ cells as $\mathrm{CD} 45^{+} \mathrm{CD}^{+} \mathrm{CD}^{+} 6^{+}, \mathrm{T}$ lymphocytes as $\mathrm{CD}^{+} 5^{+} \mathrm{CD}^{+}, \mathrm{CD}^{+} \mathrm{T}$ lymphocytes as $\mathrm{CD}_{4} 5^{+} \mathrm{CD} 3^{+} \mathrm{CD} 8{ }^{-}, \mathrm{CD}^{+} \mathrm{T}$ lymphocytes as $\mathrm{CD}^{+} 5^{+} \mathrm{CD} 3^{+} \mathrm{CD} 8^{+}$, and regulatory $\mathrm{T}$ cells as $\mathrm{CD} 45^{+} \mathrm{CD} 3^{+} \mathrm{CD} 8^{-} \mathrm{CD} 25^{++} \mathrm{CD} 127^{-}$.

\section{Induction of RGS1 gene expression with interferon-beta (IFN- $\beta$ )}

PBMC $\left(1 \times 10^{6}\right.$ cells $\left./ \mathrm{mL}\right)$ from 6 untreated MS patients (3 patients without rare variants and 3 patients carrying at least 1 rare variant in the $R G S 1$ gene) were incubated $\left(37^{\circ} \mathrm{C}\right.$, $5 \% \mathrm{CO}_{2}$ ) and grown in RPMI medium supplemented with $10 \%$ heat-inactivated fetal bovine serum (FBS), $2.0 \mathrm{mM}$ L-glutamine, 0.4\% penicillin-streptomycin and $10 \mathrm{mM}$ HEPES. Cells were incubated in the presence or absence of IFN- $\beta-1 b$ (100 International Units (IU)/mL) for 4 hours and then washed in cold PBS. Total RNA was isolated using an RNeasy ${ }^{\circledR}$ Mini Kit (Qiagen, Santa Clarita, USA) and cDNA synthesized using the High-Capacity cDNA Reverse Transcription Kit (Applied Biosystems, Foster City, CA). RGS1 mRNA expression levels were determined by real-time PCR as described above using a specific TaqMan ${ }^{\circledR}$ gene expression assay (Hs01023772_m1) and GAPDH as endogenous control (Hs02786624_g1) (Applied Biosystems). Results were expressed as fold-change in gene expression in patients with relapse-onset MS with rare variants relative to those without rare variants in the $R G S 1$ gene.

\section{Statistical analysis}

Statistical analysis was performed by using the SPSS 17.0 package (SPSS Inc, Chicago, IL) for MS-Windows. A Wilcoxon-Mann-Whitney test was used to determine differences 
in mRNA expression levels of RGS1, CYP24A1, FCRL1 and TRAF3 between PBMC from MS patients with and without rare variants within specific regions, and to test for significant differences in the percentage of PBMC subsets expressing RGS1 between MS patients with and without $R G S 1$ rare variants. A paired Student t test was applied to evaluate the induction in $R G S 1$ gene expression after IFN- $\beta$ stimulation in PBMC from MS patients with and without $R G S 1$ rare variants. p-values below 0.05 were considered statistically significant.

\section{Standard protocol approvals, registrations, and patient consents}

The study was approved by the corresponding local ethics committees, and written informed consent was obtained from all participants. 


\section{RESULTS}

\section{Targeted resequencing identified an enriched rare variants burden in specific} regions of $C Y P 24 A 1, R G S 1, F C R L 1$ and $T R A F 3$ in MS patients

Among the 2,082 detected biallelic polymorphic variants (1,903 Variants and 179 indels), $76.3 \%$ of them were found at frequencies $\leq 0.005$. To investigate whether the number of rare variants differed between MS cases and controls, we applied a set of different collapsing strategies setting a MAF threshold of 0.005 . Collectively, no significant differences were observed between cases and controls when considering jointly all the variants occurring in the whole set of genes under analysis at a frequency $\leq 0.005$ (data not shown). In contrast, the analysis of individual genes suggested the relevance of rare variants. As shown in Figure 1A, for RGS1 we observed a general enrichment of rare variants in MS cases ( 8 out of 11 collapsing methods $p<0.05,4$ out of 11 collapsing methods $\mathrm{p}<0.05$ after multiple testing correction) when considering all types of variants (i.e. coding and regulatory) together.

Previous studies demonstrated that rare variation is particularly enriched for deleterious alleles (Fu et al., 2013), suggesting that such non-common variation could have a major role in disease susceptibility (Lohmueller, 2014). Similarly to previous observations, our analysis confirms that rare variants represent the vast majority of putative functional variation in the genes under investigation. A total of $87.1 \%$ of non-synonymous variants and $92.3 \%$ of putative damaging variants were observed at a frequency $\leq 0.005$. Conversely, only $75.3 \%$ of variation located within regulatory sequences was observed at low frequency. To explore whether particular rare functional variants could contribute to MS susceptibility, we also performed the rare-variant enrichment analysis considering six different categories of putative functional variants separately (namely, coding, codealtering, putative code-damaging, regulatory, promoter and UTR). As shown in Figure 
1B, a significant rare-variant enrichment was observed in protein-coding sequences of the TIMMDC1 gene in MS patients when compared to healthy controls (1 out of 11 tests $\mathrm{p}<0.05)$. However, when restricting the analysis to code-altering variants or to putative code-damaging variants (Supplementary Table 1), no enrichment was observed neither on the whole set or in individual genes (results not shown). As depicted in Figure 1C, an enrichment of rare variants was found in the regulatory sequences of RGS1 (5 out of 11 tests $\mathrm{p}<0.05)$ and CYP24A1 (9 out of 11 tests $\mathrm{p}<0.05)$ in MS cases when compared to controls. As for the promoter region, an enrichment of rare variants was observed in the FCRL1 and CYP24A1 genes in MS cases (2 out of 11 and 6 out of 11 collapsing methods $\mathrm{p}<0.05$, respectively) versus controls (Figure 1D). Finally, the untranslated regions (UTRs) of TRAF3 (2 out of the 11 performed tests) and CYP24A1 (11 out of 11 performed tests) were also enriched for rare variants in MS cases compared to controls (Figure 1E). However, none of the rare variant enrichments observed for these specific functional categories survived multiple testing correction in any of the genes included (Figure 1B-E).

Then, we analyzed whether each statistically significant rare variants enrichment in MS cases was associated with the presence of the MS risk common variant reported in the last IMSGC large study in the same gene (7). To this end, we selected either the IMSGC associated common polymorphism or a proxy variant when the first one was not detected in our resequencing study. As shown in Supplementary Table 3 for each gene, the presence of rare variants was not associated with the presence of the selected MS-associated common variant in neither case. It should be noted that for the FCRL1 gene we did not detect neither the IMSGC associated common variant nor any applicable proxy variant in the resequencing cohort. 
mRNA expression levels of RGS1 are decreased in PBMC from MS patients with RGS1 rare variants

Based on the findings above, we next aimed to investigate whether the accumulation of rare variants was associated with changes in gene expression. For this, the expression levels of CYP24A1, FCRL1, RGS1 and TRAF3, which all showed significant enrichment for rare variants in at least two collapsing methods, were compared between patients with one or more rare variants and patients without rare variants. Of note, considering all rare variants together, mRNA expression levels for $R G S 1$ were significantly decreased in PBMC from MS patients carrying $R G S 1$ rare variants $(\mathrm{n}=6)$ compared to non-carriers $(\mathrm{n}=28)(\mathrm{p}=0.01$; Figure $2 \mathrm{~A})$. Table 1 summarizes the $R G S 1$ rare variants and their functional consequences identified in the $6 \mathrm{MS}$ patients included in the mRNA expression study. We also demonstrated that, in the $R G S 1$ gene expression cohort, the presence of $R G S 1$ rare variants was not associated with the MS-associated $R G S 1$ common variant reported in the last IMSGC meta-analysis (7) (Supplementary Table 4). Analysis of the specific accumulation of rare variants within regulatory regions only revealed a trend towards decreased $R G S 1$ expression in MS patients with rare variants compared to patients without ( $p=0.07$, Figure $2 B$ ), whereas no differences in mRNA expression levels were observed between CYP24A1 rare variants carriers and non-carriers (Figure 2B). Further analysis of sub-regulatory rare variants enrichments did not reveal significant differences in mRNA expression levels between rare variants carriers and non-carriers in the promoter region for FCRL1 and CYP24A1 (Figure 2C), and in the UTR region for TRAF3 and CYP24A1 (Figure 2D).

RGS1 expression is decreased in B lymphocytes from MS patients carrying RGS1 rare variants 
In order to identify the PBMC population responsible for the differences observed in $R G S 1$ gene expression between MS patients with and without $R G S 1$ rare variants, we next performed RGS1 immunophenotyping by flow cytometry in monocytes, B lymphocytes, $\mathrm{CD}^{+} \mathrm{T}$ lymphocytes, $\mathrm{CD} 4^{+} \mathrm{T}$ lymphocytes, $\mathrm{CD} 8^{+} \mathrm{T}$ lymphocytes, NK cells, NKT cells and regulatory T cells from 4 MS patients with one or more $R G S 1$ rare variants (Table 1 ) and 7 patients without rare variants. As shown in Figure 3, significant differences were only observed for B cells, and the percentage of B lymphocytes expressing RGS1 was significantly decreased in MS patients with $R G S 1$ rare variants compared with those patients without $(\mathrm{p}=0.006)$. These results are not affected by the presence of the RGSI common risk variant reported in the last IMSGC analysis (7) (Supplementary Table 3). Additionally, except for regulatory $\mathrm{T}$ cells, the percentage of $\mathrm{RGS1}^{+}$cells was overall decreased in monocytes, $\mathrm{CD}^{+} \mathrm{T}$ lymphocytes, $\mathrm{CD} 4^{+}$and $\mathrm{CD} 8^{+} \mathrm{T}$ lymphocytes, $\mathrm{NK}$ and NKT cells from MS patients carrying $R G S 1$ rare variants, although differences did not reach statistical significance (Figure 3).

\section{The presence of rare variants in the RGS1 gene is associated with diminished induction of $R G S 1$ gene expression by IFN- $\beta$}

As a next step, and considering that RGS1 expression can be induced by IFN- $\beta$, a widely used first line treatment for MS (Tran et al., 2010), we examined whether the accumulation of rare variants in the $R G S 1$ gene could functionally influence the capacity of IFN- $\beta$ to induce $R G S 1$ expression. PBMC from 3 MS patients with one or more RGS1 rare variants (Table 1) and 3 MS patients without rare variants were stimulated for 4 hours with IFN- $\beta$ followed by determination of $R G S 1$ mRNA expression levels by PCR, as described in Methods. As shown in Figure 4, in PBMC from MS patients without RGS1 rare variants, IFN- $\beta$ exposure was associated with a significant increase in $R G S 1$ mRNA 
expression levels $(\mathrm{p}=0.007)$. In contrast, the presence of $R G S 1$ rare variants was associated with a lack of induction of $R G S 1$ expression by the effect of IFN- $\beta$ ( $\mathrm{p}=0.31$; Figure 4). These findings are not influenced by the presence of the $R G S 1$ common risk polymorphism identified in the last IMSGC study (7) (Supplementary Table 3). Figure 5 shows the location of the RGS1 gene rare variants carried by all MS patients included in the functional studies.

\section{RGS1 rare variants enrichment and MS disease course}

We also investigated whether the accumulation of $R G S 1$ rare variants in MS patients could act as genetic modifier of the disease by analyzing their effect on age at disease onset, multiple sclerosis severity score (MSSS), \% of patients with relapsing-remitting and secondary progressive disease course, and time to reach a secondary progressive disease course in the whole resequenced cohort. As shown in Supplementary Table 3, no significant differences for these clinical variables were observed between MS patients with and without $R G S 1$ rare variants.

\section{$R G S 1$ rare variants and binding evidence in different cell types}

Finally, we investigated the experimentally measured binding evidence potentially affected by the RGS1 rare variants loci carried by the MS patients included in the functional experiments by means of variants annotation with known and predicted regulatory elements in the intergenic regions of the human genome. For each rare variant, Supplementary Table 5 shows its computed RegulomeDB score, based on the integration of multiple high-throughput datasets, as well as a set of its specific region bound proteins across many cell types. This effort demonstrates which $R G S 1$ rare variants have demonstrated potential regulatory functions and in which specific cells these effects 
would occur. Overall, we observe that a large proportion of $R G S 1$ rare variation is likely to affect regulatory function throughout various cell types. 


\section{DISCUSSION}

Although GWAS have significantly contributed to the identification of a large number of common genetic variants associated with the risk for developing MS, there is general agreement that very little is known about the role of rare genetic variants in common diseases such as MS (Cirulli \& Goldstein, 2010). The now disputed 'Common Disease, Common Variant (CDCV)' hypothesis supports that a common disease is largely attributable to a moderate number of common variants with low penetrance, and each contributes to the risk of developing MS to a certain percentage. Conversely, the most recent 'Common Disease, Rare Variant (CDRV)' hypothesis argues that highly penetrant rare variants are the main contributors to genetic susceptibility to common diseases (Schork, Murray, Frazer, \& Topol, 2009). These two paradigms are not mutually exclusive, but rather they are complementary (Gibson, 2012), motivating the rapidly growing interest of the scientific community to rare variants.

In the present study, we propose a potential contribution of rare genetic variants to the underlying genetic basis of MS. Targeted resequencing in an Iberian population led us to the identification of rare variants enrichment in specific regions of MS risk genes. In particular, whereas we found no enrichment of code-altering rare variants on the targeted MS risk genes, the coding sequences of the TIMMDC1 gene and several putatively regulatory regions on particular MS risk genes showed significant enrichment for rare variants in MS patients. To elucidate the possible regulatory effect of such rare variants accumulation in the CYP24A1, FCRL1, TRAF3 and RGS1 genes we performed several functional experiments. First, mRNA expression levels were determined in PBMC from a subgroup of MS patients with at least one rare variant in the sequence reported to be enriched. Although no significant differences in gene expression levels were observed for CYP24A1, FCRL1 and TRAF3 genes at the PBMC level, these findings do not exclude a 
potential role of the identified rare variants enrichments in MS pathogenesis, as transcriptional signatures differ even more across tissues than individuals (Melé et al., 2015). Certain genetic variants could affect gene expression not in PBMC, but in other human tissues such as brain. Unfortunately, post-mortem brain tissue samples were not available from the MS patients included in our study to explore potential gene expression differences linked to rare variants enrichment.

On the other hand, we report interesting findings in $R G S 1$, a gene previously associated with other inflammatory diseases (Hunt et al., 2008). In our study, MS patients carrying at least one rare variant in $R G S 1$ showed an overall downregulation of $R G S 1$ gene expression in PBMC compared to MS patients without rare variants, which made the RGS1 gene the main focus of our subsequent studies. The role of $R G S 1$ in MS pathogenesis had already been supported by reported genetic associations in at least three different GWAS meta-analyses. Through a meta-analysis based on a number of GWAS that included 2,624 MS patients and 7,220 healthy controls and a subsequent replication study in an independent cohort of 2,215 MS subjects and 2,116 controls, $R G S 1$ was first unmasked as a novel locus with putative evidence of association to MS (rs2760524, $\left.p=9.77 \times 10^{-6}\right)($ De Jager et al., 2009). Based on this finding, the International Multiple Sclerosis Genetics Consortium (IMSGC) aimed to validate the new MS susceptibility polymorphism by genotyping rs 2760524 in additional sample sets $(8,085 \mathrm{MS}$ patients and 7,777 healthy controls) and then combining the new results with those of the published meta-analysis and its replication study. In this case, rs2760524 was found to be associated to MS with evidence of association at the genome-wide level of significance $\left(p=3.55 \times 10^{-}\right.$ ${ }^{9}$ ) (Esposito et al., 2010). Subsequently, another MS susceptibility variant around RGS1 and in strong linkage disequilibrium with the previously reported variant $\left(r^{2}=0.943\right.$; $\mathrm{D}^{\prime}=1.000$ ) was detected in a collaborative GWAS involving 9,772 MS cases and 17,376 
healthy controls of European origin ( $\mathrm{rs} 1323292, \mathrm{p}=8.80 \times 10^{-7}$ ) (Sawcer et al., 2011), and then recently validated in a large meta-analysis $\left(p=3.64 \mathrm{E}^{-33}\right)$ (Patsopoulos et al., 2019). Through the ImmunoChip custom genotyping array, the IMSGC tested 161,311 variants on 38,589 individuals of European ancestry (14,498 MS subjects, 24,091 healthy controls) and then combined these data with previous GWAS data from 14,802 MS patients and 26,703 healthy controls of European ancestry. In these 80,094 individuals, they identified a third variant in RGS1 associated with MS at the genome-wide significance level (rs1359062, $\mathrm{p}=1.8 \times 10^{-13}$ ) (Beecham et al., 2013), also in linkage disequilibrium with the previously reported two polymorphisms $\left(\mathrm{r}^{2}=0.891 ; \mathrm{D}^{\prime}=1.000\right.$ and $\mathrm{r}^{2}=0.945 ; \mathrm{D}^{\prime}=1.000$, respectively). To date, there was no evidence of association of RGS1 rare variants with MS.

RGS1 is expressed in lymphocytes, dendritic cells, monocytes (Bansal, Druey, \& Xie, 2007) and microglia (Atwood, Lopez, Wager-Miller, Mackie, \& Straiker, 2011). It has been reported that B lymphocytes in germinal centers (GC) within follicles of lymphoid organs express specially high levels of RGS1 (C Moratz et al., 2000), which codes for the Regulator of G-protein signalling 1, a protein located on the cytosolic side of the plasma membrane that acts as a negative regulator of G-protein-coupled receptors (GPCRs) signalling (Lee \& Bou Dagher, 2016). The fact that all chemokine receptors are coupled to heterotrimeric G-proteins (Ansel \& Cyster, 2001) promoted the investigation of the role of RGS1 in the response to chemokine stimulation and led to uncover that B cells in RGS1-/- mice respond extremely to the chemokines CXCL12 (CXCR4 ligand) and CXCL13 (CXCR5 ligand) (Chantal Moratz, Hayman, Gu, \& Kehrl, 2004). Interestingly, tracking of $R G S 1-/-$ and wild type B cells adoptively transferred into experimental mice revealed that the lack of $R G S 1$ boosts the entrance of B cells into peripheral lymph nodes 
and enhances their motility within follicles (Han et al., 2005). Our finding that the number of peripheral B cells lacking RGS1 is increased in MS patients with RGS1 rare variants in comparison with those without may result in their faster entrance into lymph nodes follicles, also in accordance with the finding that the number of intravenously transferred $R G S 1^{-1-} \mathrm{B}$ cells is decreased relative to wild type cells in mice peripheral blood (Han et al., 2005). In this regard, we hypothesize that the defect in RGS1 expression by B cells of MS patients with $R G S 1$ rare variants could foster a more active phenotype of such cells and therefore eases their ability to migrate across the blood-brain barrier (BBB) and access the CNS. Other reported cell response to chemokine stimulation is the increase in intracellular calcium (Turner, Nedjai, Hurst, \& Pennington, 2014). In this context, Moratz et al. observed an increased rise in intracellular $\mathrm{Ca}^{2+}$ in follicular $R G S 1^{-1-} \mathrm{B}$ cells when compared to wild type B cells after their exposure to CXCL12 (Chantal Moratz et al., 2004), which is in agreement with the exacerbated response of $R G S 1^{-/-}$B cells to chemokines. It has also been reported that an increase in intracellular $\mathrm{Ca}^{2+}$ levels activates other signalling pathways that are critical in determining both the $\mathrm{B}$ cell fate during humoral immune responses (Scharenberg, Humphries, \& Rawlings, 2007) and processes such as cell proliferation and differentiation(Izquierdo, Bonilla-Abadía, Cañas, \& Tobón, 2014). Regarding our study, B cells from MS patients carrying $R G S 1$ rare variants would be supposed to show an overstated raise in intracellular $\mathrm{Ca}^{2+}$ levels following CXCL12 exposure, so their immune reaction could be aberrant. Additionally, the altered proliferation of autoreactive B cells in these MS patients may result in a higher secretion of antibodies to self-antigens.

Another relationship between the RGS1 gene and MS emerges from its previously reported increased mRNA levels in PBMC from MS patients following IFN- $\beta$ treatment 
(Tran et al., 2010). As shown by our results, PBMC from MS patients lacking RGS1 rare variants exhibited the expected induction of $R G S 1$ gene expression after IFN- $\beta$ stimulation. Conversely, the presence of $R G S 1$ rare variants was associated with functional consequences insomuch as $R G S 1$ expression was not induced by IFN- $\beta$ treatment. Whether this lack of induction in $R G S 1$ gene expression by IFN- $\beta$ is associated with a lack of response to treatment is unknown and merits further investigation.

In conclusion, we observed that MS patients tend to accumulate rare variants of the RGS1 gene when compared with healthy controls. Our data suggest that rare variants accumulation is associated with functional consequences such as decreased $R G S 1$ mRNA expression levels in PBMC, reduced percentage of B lymphocytes expressing RGS1 and lack of induction in $R G S 1$ gene expression by IFN- $\beta$.

\section{ACKNOWLEDGEMENTS}

This work was supported by grants from the Fondo de Investigación Sanitaria (FIS; grant number PI12/02229; Ministry of Science and Innovation, Spain), Agencia Estatal de Investigación (AEI) and Fondo Europeo de Desarollo Regional (FEDER; grant number BFU2016-77961-P), Direcció General de Recerca - Generalitat de Catalunya (2017-SGR00702), Unidad de Excelencia María de Maeztu (MINECO; MDM-2014-0370), and Vall d'Hebrón Institut de Recerca (predoctoral grants programme 2014). 


\section{ARTICLE INFORMATION}

\section{Contributorship}

EGV: manuscript drafting and revision, study concept and design, peripheral blood expression studies, interferon-beta experiments, statistical analysis, and interpretation of the data.

NS: manuscript drafting and revision, study concept and design, bioinformatics analysis, statistical analysis, and interpretation of the data.

LMV, ATV: manuscript revision, immunophenotyping experiments, analysis, and interpretation of the data.

LM: manuscript revision, clinical data collection, analysis, and interpretation of the data. FM: manuscript drafting and revision, study concept and design, statistical analysis, and interpretation of the data.

SM: manuscript revision, peripheral blood expression studies, analysis, and interpretation of the data.

HE: manuscript revision, immunophenotyping experiments design, analysis, and interpretation of the data.

NP: manuscript drafting and revision, study concept and design, analysis, and interpretation of the data.

OF, BOM, AS, SL, LRT, EQ, GI, AA: manuscript revision, contribution of patients and clinical data, analysis, and interpretation of the data.

EB, AN: manuscript drafting and revision, study concept and design, bioinformatics analysis, statistical analysis, and interpretation of the data.

XM: manuscript revision, contribution of patients and clinical data, analysis, and interpretation of the data. 
MC: manuscript drafting and revision, study concept and design, analysis and interpretation of the data, study supervision.

All authors read and approved the final manuscript.

\section{Conflict of Interest Disclosures}

There are no competing interests for any author.

\section{Data Availability Statement}

The data that support the findings of this study are available from the corresponding author upon reasonable request. Variant sequence data for novel variants presented in Table 1 and Supplementary Table 1 have been submitted to dbSNP under accession numbers ss 2137544070 and ss3943621973 to ss394362200. 


\section{REFERENCES}

Alcina, A., Fedetz, M., Fernández, O., Saiz, A., Izquierdo, G., Lucas, M... Matesanz, F. (2013). Identification of a functional variant in the KIF5A-CYP27B1-METTL1FAM119B locus associated with multiple sclerosis. J Med Genet, 50(1), 25-33. https://doi.org/10.1136/jmedgenet-2012-101085.

Ansel, K. M., \& Cyster, J. G. (2001). Chemokines in lymphopoiesis and lymphoid organ development. Current Opinion in Immunology, 13(2), 172-179. Alcina, A., Fedetz, M., Fernández, O., Saiz, A., Izquierdo, G., Lucas, M... Matesanz, F. (2013). Identification of a functional variant in the KIF5A-CYP27B1-METTL1FAM119B locus associated with multiple sclerosis. J Med Genet, 50(1), 25-33. https://doi.org/10.1136/jmedgenet-2012-101085.

Ascherio, A. (2013). Environmental factors in multiple sclerosis. Expert Review of Neurotherapeutics, 13(sup2), 3-9. https://doi.org/10.1586/14737175.2013.865866

Atwood, B. K., Lopez, J., Wager-Miller, J., Mackie, K., \& Straiker, A. (2011). Expression of $\mathrm{G}$ protein-coupled receptors and related proteins in HEK293, AtT20, BV2, and N18 cell lines as revealed by microarray analysis. BMC Genomics, 12, 14. https://doi.org/10.1186/1471-2164-12-14

Bansal, G., Druey, K. M., \& Xie, Z. (2007). R4 RGS proteins: Regulation of G-protein signaling and beyond. Pharmacology \& Therapeutics, 116(3), 473-495. https://doi.org/10.1016/j.pharmthera.2007.09.005

Beecham, A. H., Patsopoulos, N. A., Xifara, D. K., Davis, M. F., Kemppinen, A., Cotsapas, C., ... McCauley, J. L. (2013). Analysis of immune-related loci identifies 48 new susceptibility variants for multiple sclerosis. Nature Genetics, 45(11), 1353-1360. https://doi.org/10.1038/ng.2770

Benveniste, E. N., Liu, Y., McFarland, B. C., Qin, H. (2014). Involvement of the janus kinase/signal transducer and activator of transcription signaling pathway in multiple sclerosis and the animal model of experimental autoimmune encephalomyelitis. J Interferon Cytokine Res. 34(8):577-88. https://doi.10.1089/jir.2014.0012. 
Chen D, Ireland SJ, Remington G, Alvarez E, Racke MK, Greenberg B, Frohman EM, Monson NL.(2016). CD40-Mediated NF- $\kappa$ B Activation in B Cells Is Increased in Multiple Sclerosis and Modulated by Therapeutics. J Immunol. 197(11):42574265. https://doi.org/10.4049/jimmunol.1600782.

Cirulli, E. T., \& Goldstein, D. B. (2010). Uncovering the roles of rare variants in common disease through whole-genome sequencing. Nature Reviews Genetics, 11(6), 415-425. https://doi.org/10.1038/nrg2779

Comabella, M., Cantó, E., Nurtdinov, R., Río, J., Villar, L. M., Picón, C., Castilló, J., Fissolo, N., Aymerich, X., Auger, C., Rovira, A.,... Montalban, X. (2016). MRI phenotypes with high neurodegeneration are associated with peripheral blood Bcell changes. Hum Mol Genet, 25(2), 308-16. https://doi.org/10.1093/hmg/ddv473.

De Jager, P. L., Jia, X., Wang, J., de Bakker, P. I. W., Ottoboni, L., Aggarwal, N. T., ... Oksenberg, J. R. (2009). Meta-analysis of genome scans and replication identify CD6, IRF8 and TNFRSF1A as new multiple sclerosis susceptibility loci. Nature Genetics, 41(7), 776-782. https://doi.org/10.1038/ng.401

Ebers, G. C. (2008). Environmental factors and multiple sclerosis. The Lancet Neurology, 7(3), 268-277. https://doi.org/10.1016/S1474-4422(08)70042-5

Esposito F., Patsopoulos N.A., Cepok S., Kockum I., Leppä V., Booth D.R., ... De Jager P.L. (2010). IL12A, MPHOSPH9/CDK2AP1 and RGS1 are novel multiple sclerosis susceptibility loci. Genes \& Immunity, 11(5), 397-405. https://doi.org/10.1038/gene.2010.28

Fu, W., O’Connor, T. D., Jun, G., Kang, H. M., Abecasis, G., Leal, S. M., ... Akey, J. M. (2013). Analysis of 6,515 exomes reveals the recent origin of most human proteincoding variants. Nature. https://doi.org/10.1038/nature11690

Gibson, G. (2012). Rare and common variants: twenty arguments. Nature Reviews Genetics, 13(2), 135-145. https://doi.org/10.1038/nrg3118

Han, S. B., Moratz, C., Huang, N. N., Kelsall, B., Cho, H., Shi, C. S., ... Kehrl, J. H. (2005). Rgs1 and Gnai2 regulate the entrance of B lymphocytes into lymph nodes and B cell motility within lymph node follicles. Immunity. https://doi.org/10.1016/j.immuni.2005.01.017 
Hunt, K. A., Zhernakova, A., Turner, G., Heap, G. A. R., Franke, L., Bruinenberg, M., ... van Heel, D. A. (2008). Newly identified genetic risk variants for celiac disease related to the immune response. Nature Genetics, 40(4), 395-402. https://doi.org/10.1038/ng.102

Inoue, M., Chen, P.H,, Siecinski, S., Li, Q.J., Liu, C., Steinman, L. (2016). An interferon$\beta$-resistant and NLRP3 inflammasome-independent subtype of EAE with neuronal damage. Nat Neurosci. 19(12):1599-1609. https://doi: 10.1038/nn.4421.

Izquierdo, J.-H., Bonilla-Abadía, F., Cañas, C. A., \& Tobón, G. J. (2014). Calcio, canales, señalización intracelular y autoinmunidad. Reumatología Clínica, 10(1), 43-47. https://doi.org/10.1016/j.reuma.2013.05.008

Kakalacheva, K., \& Lünemann, J. D. (2011). Environmental triggers of multiple sclerosis. FEBS Letters. https://doi.org/10.1016/j.febslet.2011.04.006

Klimatcheva, E., Pandina, T., Reilly, C., Torno, S., Bussler, H., Scrivens, M... Zauderer M. (2015). BMC Immunol. 16,6. https://doi.org/10.1186/s12865-015-0068-1

Lee, J.-K., \& Bou Dagher, J. (2016). Regulator of G-protein Signaling (RGS)1 and RGS10 Proteins as Potential Drug Targets for Neuroinflammatory and Neurodegenerative Diseases. The AAPS Journal, 18(3), 545-549. https://doi.org/10.1208/s12248-016-9883-4

Lettre, G. (2014). Rare and low-frequency variants in human common diseases and other complex traits. Journal of Medical Genetics. https://doi.org/10.1136/jmedgenet-2014-102437

Livak, K. J., \& Schmittgen, T. D. (2001). Analysis of relative gene expression data using real-time quantitative PCR and the 2- $\Delta \Delta \mathrm{CT}$ method. Methods, 25(4), 402408. https://doi.org/10.1006/meth.2001.1262

Lohmueller, K. E. (2014). The Impact of Population Demography and Selection on the Genetic Architecture of Complex Traits. PLoS Genetics, 10(5), e1004379. https://doi.org/10.1371/journal.pgen.1004379

Malmeström, C., Gillett, A., Jernås, M., Khademi, M., Axelsson, M., Kockum, I...Olsson B. (2012). Serum levels of LIGHT in MS. Mult Scler. 19(7), 871-6. 
https://doi: 10.1177/1352458512463766.

Manolio, T. A., Collins, F. S., Cox, N. J., Goldstein, D. B., Hindorff, L. A., Hunter, D. J., ... Visscher, P. M. (2009). Finding the missing heritability of complex diseases. Nature, 461(7265), 747-753. https://doi.org/10.1038/nature08494

Melé, M., Ferreira, P. G., Reverter, F., DeLuca, D. S., Monlong, J., Sammeth, M., ... Guigó, R. (2015). The human transcriptome across tissues and individuals. Science. https://doi.org/10.1126/science.aaa0355

Mitrovič M., Patsopoulos N.A., Beecham A.H., Dankowski T., Goris A., Dubois B., ... Cotsapas, C. (2018). Low-Frequency and Rare-Coding Variation Contributes to Multiple Sclerosis Risk. Cell. https://doi.org/10.1016/j.cell.2018.09.049

Moratz, C., Hayman, J. R., Gu, H., \& Kehrl, J. H. (2004). Abnormal B-Cell Responses to Chemokines, Disturbed Plasma Cell Localization, and Distorted Immune Tissue Architecture in Rgs1-/- Mice. Molecular and Cellular Biology, 24(13), 5767-5775. https://doi.org/10.1128/MCB.24.13.5767-5775.2004

Moratz, C., Kang, V. H., Druey, K. M., Shi, C. S., Scheschonka, A., Murphy, P. M., ... Kehrl, J. H. (2000). Regulator of G protein signaling 1 (RGS1) markedly impairs Gi alpha signaling responses of B lymphocytes. Journal of Immunology,f 164(4), 1829-1838. https://doi.org/10.4049/jimmunol.164.4.1829

Oksenberg, J. R. (2013). Decoding multiple sclerosis: an update on genomics and future directions. Expert Review of Neurotherapeutics, 13(sup2), 11-19. https://doi.org/10.1586/14737175.2013.865867

Patsopoulos, N., Baranzini, S. E., Santaniello, A., Shoostari, P., Cotsapas, C., Wong, G., ... De Jager, P. L. (2019). The Multiple Sclerosis Genomic Map: role of peripheral immune cells and resident microglia in susceptibility. Science. 365, 6460. https://doi.org/10.1126/science.aav7188.

Ramasamy, A., Trabzuni, D., Forabosco, P., Smith, C., Walker, R., Dillman, A. (2014). Genetic evidence for a pathogenic role for the vitamin D3 metabolizing enzyme CYP24A1 in multiple sclerosis. Mult Scler Relat Disord. 3(2), 211-219.

Saint Pierre, A., \& Genin, E. (2014). How important are rare variants in common 
disease? Briefings in Functional Genomics, 13(5), 353-361.

https://doi.org/10.1093/bfgp/elu025

Sawcer, S., Hellenthal, G., Pirinen, M., Spencer, C. C. A., Patsopoulos, N. A., Moutsianas, L., ... Compston, A. (2011). Genetic risk and a primary role for cellmediated immune mechanisms in multiple sclerosis. Nature, 476(7359), 214-219. https://doi.org/10.1038/nature10251

Scharenberg, A. M., Humphries, L. A., \& Rawlings, D. J. (2007). Calcium signalling and cell-fate choice in B cells. Nature Reviews. Immunology, 7(10), 778-789. https://doi.org/10.1038/nri2172

Schork, N. J., Murray, S. S., Frazer, K. A., \& Topol, E. J. (2009). Common vs. rare allele hypotheses for complex diseases. Current Opinion in Genetics \& Development, 19(3), 212-219. https://doi.org/10.1016/j.gde.2009.04.010.

Scott-Hewitt, N.J., Folts, C.J., Hogestyn, J.M;, Piester, G; Mayer-Pröschel, M; Noble, M.D. (2017). Heterozygote galactocerebrosidase (GALC) mutants have reduced remyelination and impaired myelin debris clearance following demyelinating injury. Hum Mol Genet.26(15), 2825-2837. https://doi.org/10.1093/hmg/ddx153.

Thompson, A. J., Banwell, B. L., Barkhof, F., Carroll, W. M., Coetzee, T., Comi, G., ... Cohen, J. A. (2018). Diagnosis of multiple sclerosis: 2017 revisions of the McDonald criteria. The Lancet Neurology, 17(2), 162-173. https://doi.org/10.1016/S1474-4422(17)30470-2

Tran, T., Paz, P., Velichko, S., Cifrese, J., Belur, P., Yamaguchi, K. D., ... Croze, E. (2010). Interferon $\beta-1 b$ induces the expression of RGS1 a negative regulator of Gprotein signaling. International Journal of Cell Biology, 2010, 529376. https://doi.org/10.1155/2010/529376

Turner, M. D., Nedjai, B., Hurst, T., \& Pennington, D. J. (2014, November). Cytokines and chemokines: At the crossroads of cell signalling and inflammatory disease. Biochimica et Biophysica Acta. Netherlands. https://doi.org/10.1016/j.bbamcr.2014.05.014

Wang, K., Li, M., \& Hakonarson, H. (2010). ANNOVAR: functional annotation of genetic variants from high-throughput sequencing data. Nucleic Acids Research, 
38(16), e164-e164. https://doi.org/10.1093/nar/gkq603.

West, L. C., Cresswell, P. (2013). Expanding roles for GILT in immunity. Curr Opin Immunol, 25(1), 103-8. https://doi: 10.1016/j.coi.2012.11.006. 


\section{FIGURE LEGENDS}

\section{Figure 1. Manhattan plots of rare variants association results.}

A total of 11 collapsing methods were applied to test whether rare variants in MS patients and healthy controls are differently distributed within specific genomic regions of the 14 selected genes under analysis. (A) Analysis of rare variants enrichment taking into account all variants categories. (B) Analysis of rare variants accumulation counting only those located within protein-coding sequence of genes. (C) Analysis of rare variants enrichment considering only those located within regulatory sequences. (D) Analysis of rare variants accumulation within the promoter region of genes. (E) Analysis of rare variants enrichment in untranslated regions (UTR). All plots show - $\log _{10}$ (p-value) on the $y$ axis and the 14 included genes on the $x$ axis. The black horizontal lines correspond to $\mathrm{p}$-value $=0.05$ and the red horizontal lines to $\mathrm{p}$-value after Bonferroni multiple testing correction. Each symbol corresponds to one collapsing test. Rare variants enrichment in MS patients and healthy controls is represented by triangles and diamonds, respectively. Yellow symbols indicate collapsing methods significant at 0.05 level, dark red symbols represent collapsing methods significant after multiple testing correction, and grey symbols collapsing methods resulting in a significance level $\geq 0.05$.

Figure 2. Baseline expression levels of genes accumulating rare variants within specific regions in MS patients.

mRNA expression levels of genes determined to be enriched for rare variants in MS patients by at least two collapsing tests were measured by real-time PCR relative quantification in PBMC from MS patients stratified according to the presence or absence of rare variants within one of the following genomic regions: (A) the whole gene sequence, (B) regulatory sequences, (C) the promoter region or (D) the untranslated 
regions at the 5' (5'UTRs) and at the 3' (3'UTRs) of genes. Significant p-values are shown in bold. Results are expressed as fold-change in gene expression in rare variants carriers relative to non-carriers. RV: rare variants.

Figure 3. RGS1 expression in PBMC subpopulations from MS patients with and without $R G S 1$ rare variants.

Box plots showing the percentage of RGS1+ cells determined by flow cytometry in B lymphocytes, $\mathrm{CD}^{+} \mathrm{T}$ lymphocytes, $\mathrm{CD}^{+} \mathrm{T}$ lymphocytes, $\mathrm{CD} 8^{+} \mathrm{T}$ lymphocytes, $\mathrm{T}$ regulatory cells, monocytes, NK cells and NKT cells from MS patients with and without $R G S 1$ rare variants within the whole gene sequence. Significant $\mathrm{p}$-values are shown in bold. RV: rare variants.

Figure 4. RGS1 gene expression levels before and after IFN- $\beta$ induction in PBMC from MS patients.

MS patients are grouped based on the presence or absence of RGS1 rare variants. Bar graphs showing the mRNA expression levels for $R G S 1$ determined by real-time PCR in PBMC from MS patients with $(\mathrm{n}=3)$ and without $(\mathrm{n}=3) R G S 1$ rare variants in basal conditions and after 4 hours of IFN- $\beta$ treatment. Significant $p$-values are shown in bold. Data are expressed as fold-change in RGS1 gene expression in IFN- $\beta$-stimulated PBMC relative to the untreated condition, both in rare variants carriers and non-carriers. RV: rare variants.

Figure 5. Localization of rare variants at the RGS1 locus.

Schematic illustration to show the localization of the rare variants (in green). RGS MS indicates the variants for each of the 8 patients with rare variants in the locus. The 
potential regulatory regions as indicated by ENCODE are located at the bottom: Layered $H 3 \mathrm{~K} 4 \mathrm{Me} 1$ track shows where modification of histone proteins is suggestive of enhancer; Layered H3K4Me3 track shows a histone mark associated with promoters; Layered $H 3 K 4 M e 3$ track shows histone mark associated with promoters that are active or poised to be activated; $T F$ ChIP-seq track shows DNA regions where transcription factors bind to DNA as assayed by chromatin immunoprecipitation (ChIP) with antibodies specific to the transcription factor followed by sequencing of the precipitated DNA (ChIP-Seq). 
Table 1. Description of the rare RGS1 variants identified in the MS patients selected for the functional studies.

\begin{tabular}{|c|c|c|c|c|c|c|c|c|}
\hline $\begin{array}{l}\text { MS } \\
\text { patients }\end{array}$ & $\begin{array}{c}\text { Gene } \\
\text { expression }\end{array}$ & $\begin{array}{c}\text { Flow } \\
\text { cytometry }\end{array}$ & $\begin{array}{c}\text { IFN-B } \\
\text { induction }\end{array}$ & SNP (Ref./Alt.) & Location ( HGVShg19) & $\mathbf{M A F}^{\dagger}$ & $\operatorname{gnomAD}$ MAF* & $\begin{array}{l}\text { Most severe consequence in Ensembl-ACMG } \\
\text { classification }\end{array}$ \\
\hline \multirow{7}{*}{ MS1 } & \multirow{7}{*}{$\mathrm{x}$} & \multirow{7}{*}{$\mathrm{x}$} & \multirow{7}{*}{-} & rs12065772 (C/T) & NC_000001.10:g.192550461C >T & $0.005(\mathrm{~T})$ & $0.0003890(\mathrm{~T})$ & downstream gene variant-N/A \\
\hline & & & & rs142034366 & NM_002922.3:c.138-165_138- & $0.004(\mathrm{~T})$ & $0.0003243(\mathrm{~T})$ & non coding transcript exon variant-Benign \\
\hline & & & & (TAGG/T) & 163delAGG & & & \\
\hline & & & & rs74130688 (G/A) & NM_002922.3:c.-1921G>A & $0.004(\mathrm{~A})$ & $0.0002593(\mathrm{~A})$ & upstream gene variant-N/A \\
\hline & & & & rs4658058 (T/C) & NM_002922.3:c.*478T $>C$ & $0.003(\mathrm{C})$ & $0.0001296(\mathrm{C})$ & 3 prime UTR variant-Benign \\
\hline & & & & rs60719978 (C/T) & NM_002922.3:c.445-22C $>$ T & $0.003(\mathrm{~T})$ & $0.0004164(\mathrm{~T})$ & intron variant-Benign \\
\hline & & & & rs12094893 (G/A) & NC_000001.10:g.192549871G>A & $0.003(\mathrm{~A})$ & $0.0001296(\mathrm{~A})$ & downstream gene variant-N/A \\
\hline MS2 & $\mathrm{x}$ & $\mathrm{x}$ & - & ss3943621974 (G/C) & NC_000001.10:g.192550276G $>C$ & $0.0005(\mathrm{C})$ & N/A & intergenic variant-N/A \\
\hline \multirow{2}{*}{ MS3 } & \multirow{2}{*}{$\mathrm{x}$} & \multirow{2}{*}{$x$} & \multirow{2}{*}{ - } & rs16834436 (A/G) & NM_002922.3:c.280+390A $>$ G & $0.003(\mathrm{G})$ & $0.003959(\mathrm{G})$ & non coding transcript exon variant-Benign \\
\hline & & & & rs77617886 (T/A) & NM_002922.3:c.280+612T $>$ A & $0.003(\mathrm{~A})$ & $0.003372(\mathrm{~A})$ & non coding transcript exon variant-Benign \\
\hline \multirow{2}{*}{ MS4 } & \multirow{2}{*}{$\mathrm{x}$} & \multirow{2}{*}{$x$} & \multirow{2}{*}{$\mathrm{x}$} & rs74130689 (G/A) & NM_002922.3:c.*342G>A & $0.001(\mathrm{~A})$ & $0.00006485(\mathrm{~A})$ & 3 prime UTR variant-Benign \\
\hline & & & & rs12065772 (C/T) & NC_000001.10:g.192550461C > T & $0.005(\mathrm{~T})$ & $0.0003890(\mathrm{~T})$ & downstream gene variant-N/A \\
\hline \multirow{10}{*}{ MS5 } & \multirow{10}{*}{$\mathrm{x}$} & \multirow{10}{*}{ - } & \multirow{10}{*}{ - } & rs12065772 (C/T) & NC_000001.10:g.192550461C $>\mathrm{T}$ & $0.005(\mathrm{~T})$ & $0.0003890(\mathrm{~T})$ & downstream gene variant-N/A \\
\hline & & & & rs142034366 & NM_002922.3:c.138-165_138- & $0.004(\mathrm{~T})$ & $0.0003243(\mathrm{~T})$ & non coding transcript exon variant-Benign \\
\hline & & & & $(\mathrm{TAGG} / \mathrm{T})$ & 163delAGG & & & \\
\hline & & & & rs74130688 (G/A) & NM_002922.3:c.-1921G $>$ A & $0.004(\mathrm{~A})$ & $0.0002593(\mathrm{~A})$ & upstream gene variant-N/A \\
\hline & & & & rs4658058 (T/C) & NM_002922.3:c.*478T $>C$ & $0.003(\mathrm{C})$ & $0.0001296(\mathrm{C})$ & 3 prime UTR variant-Benign \\
\hline & & & & rs60719978 (C/T) & NM_002922.3:c.445-22C >T & $0.003(\mathrm{~T})$ & $0.0004164(\mathrm{~T})$ & intron variant-Benign \\
\hline & & & & rs12094893 (G/A) & NC_000001.10:g.192549871G>A & $0.003(\mathrm{~A})$ & $0.0001296(\mathrm{~A})$ & downstream gene variant-N/A \\
\hline & & & & rs3806353 (C/T) & NM_002922.3:c.-1300C $>$ T & $0.004(\mathrm{~T})$ & $0.002530(\mathrm{~T})$ & upstream gene variant-N/A \\
\hline & & & & rs3806354 (A/C) & NM_002922.3:c.-1410A>C & $0.004(\mathrm{C})$ & $0.002530(\mathrm{C})$ & upstream gene variant-N/A \\
\hline & & & & rs76219467 (A/T) & NM_002922.3:c.280+464A>T & $0.003(\mathrm{~T})$ & $0.0005188(\mathrm{~T})$ & non coding transcript exon variant-Likely Benign \\
\hline
\end{tabular}




\begin{tabular}{|c|c|c|c|c|c|c|c|c|}
\hline $\begin{array}{l}\text { MS } \\
\text { patients }\end{array}$ & $\begin{array}{c}\text { Gene } \\
\text { expression }\end{array}$ & $\begin{array}{c}\text { Flow } \\
\text { cytometry }\end{array}$ & $\begin{array}{c}\text { IFN- } \beta \\
\text { induction }\end{array}$ & SNP (Ref./Alt.) & Location (hg19) & $\mathbf{M A F} \mathbf{F}^{\dagger}$ & gnomAD MAF* & Most severe consequence \\
\hline \multirow{4}{*}{ MS6 } & \multirow{4}{*}{$\mathrm{x}$} & \multirow{4}{*}{-} & \multirow{4}{*}{-} & rs12065772 (C/T) & NC_000001.10:g.192550461C>T & $0.005(\mathrm{~T})$ & $0.0003890(\mathrm{~T})$ & downstream gene variant-N/A \\
\hline & & & & rs60719978 (C/T) & NM_002922.3:c.445-22C $>\mathrm{T}$ & $0.003(\mathrm{~T})$ & $0.0004164(\mathrm{~T})$ & intron variant-Benign \\
\hline & & & & rs113090598 (T/A) & NM_002922.3:c.*9T $>$ A & $0.0005(\mathrm{~A})$ & $0.000007835(\mathrm{~A})$ & 3 prime UTR variant-Uncertain Significance \\
\hline & & & & rs149919199 (G/C) & NM_002922.3:c.*1183G>C & $0.0005(\mathrm{C})$ & $0.000(\mathrm{C})$ & downstream gene variant-Uncertain Significance \\
\hline \multirow{2}{*}{ MS7 } & \multirow{2}{*}{-} & \multirow{2}{*}{ - } & \multirow[b]{2}{*}{$\mathrm{x}$} & rs185918389 (T/A) & NC_000001.10:g.192550105T $>A$ & $0.0009(\mathrm{~A})$ & $0.001037(\mathrm{~A})$ & downstream gene variant-N/A \\
\hline & & & & $\underline{\operatorname{rs} 370697657}(\mathrm{G} / \mathrm{A})$ & NM_002922.3:c.281-277G $>$ A & $0.001(\mathrm{~A})$ & N/A & intron variant-N/A \\
\hline MS8 & - & - & $\mathrm{x}$ & $\underline{\mathrm{rs} 370697657}(\mathrm{G} / \mathrm{A})$ & NC_000001.10:g.192549677G>A & $0.001(\mathrm{~A})$ & $\mathrm{N} / \mathrm{A}$ & downstream gene variant-N/A \\
\hline
\end{tabular}

The prefix "MS" followed by a number indicates each included MS patient carrying RGS1 rare variants. Ref.: reference allele. Alt: alternative allele. MAF: minor allele frequency. ${ }^{\dagger}$ Within the resequencing cohort. ${ }^{\dagger}$ In European (non-Finnish) populations, extracted from The Genome Aggregation Database. ACMG: American College of Medical Genetics. 


\section{Figure 1}

A

AllVariants

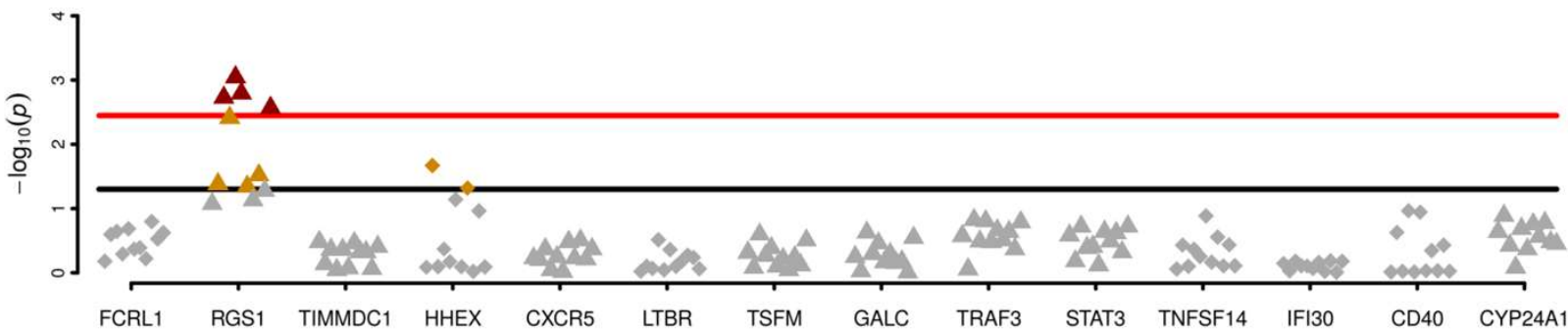

B
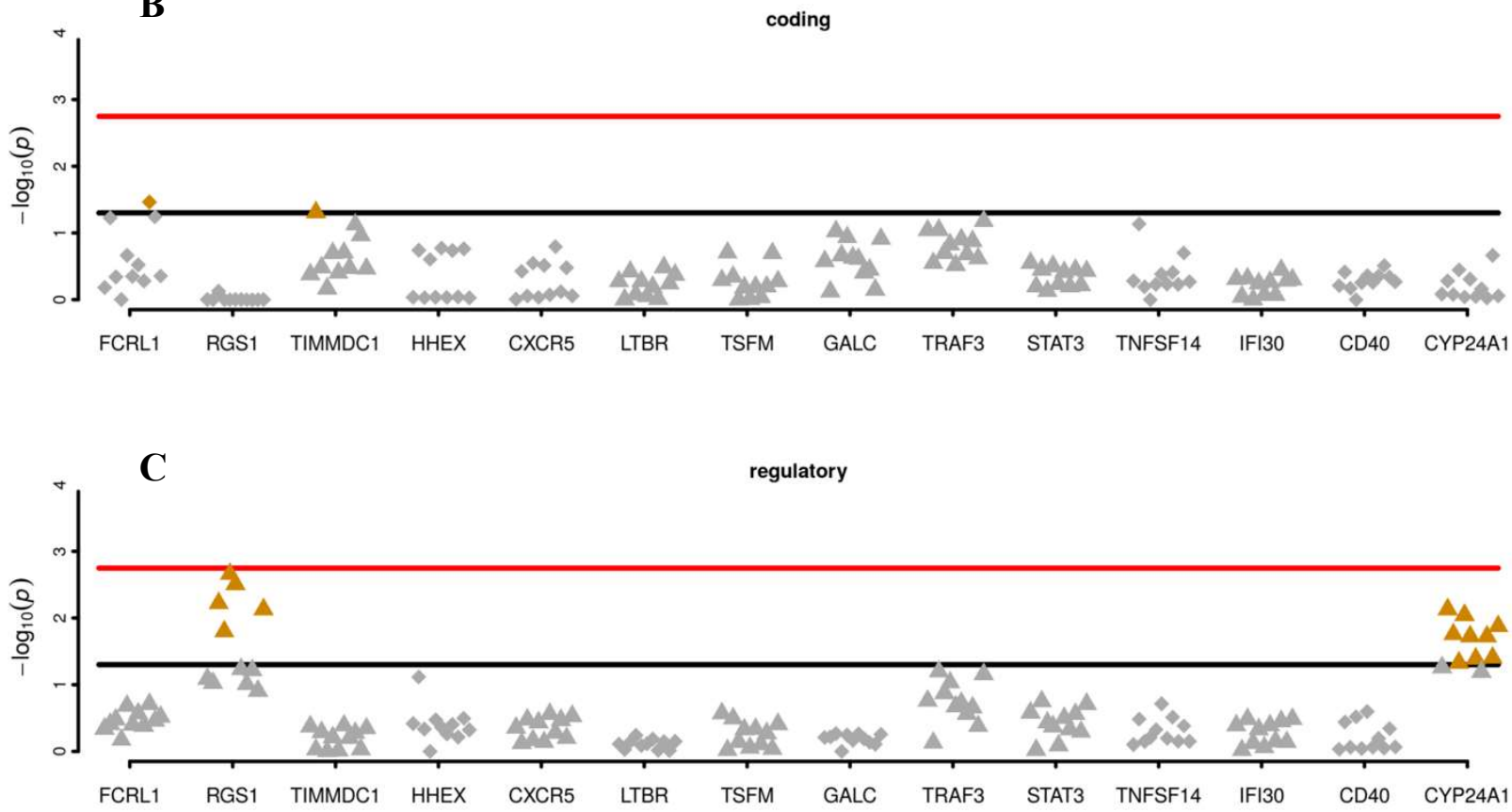

D

promoter

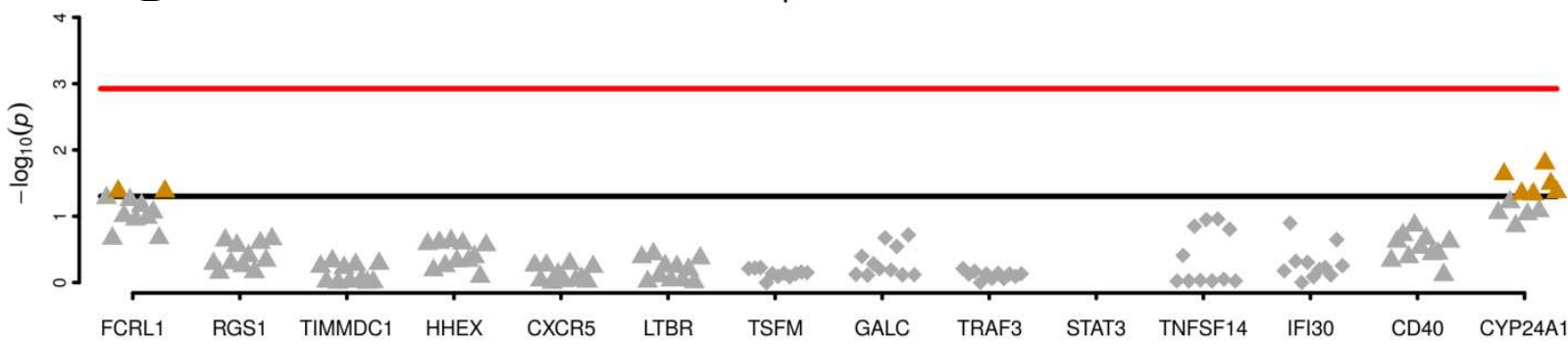

E

UTR

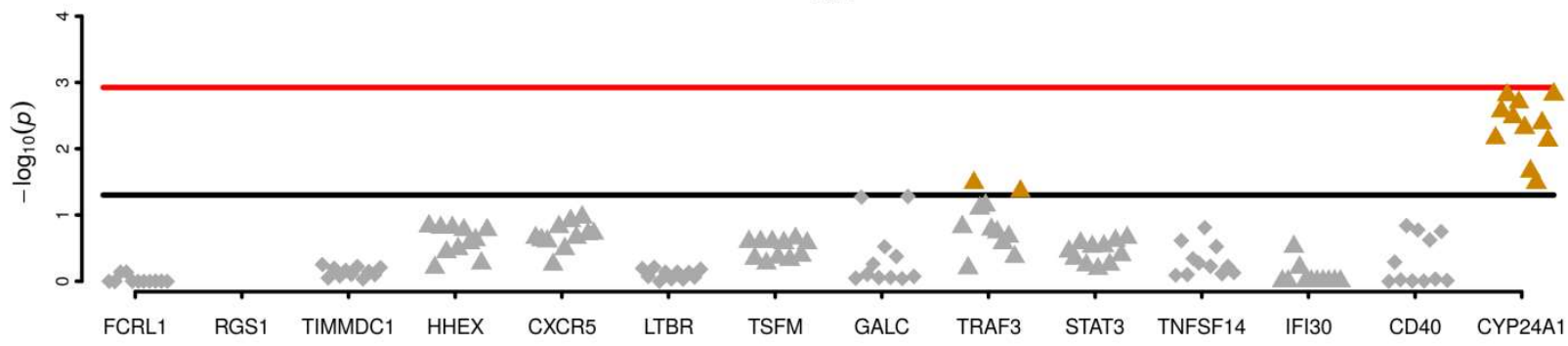


Figure 2

A. All rare variants

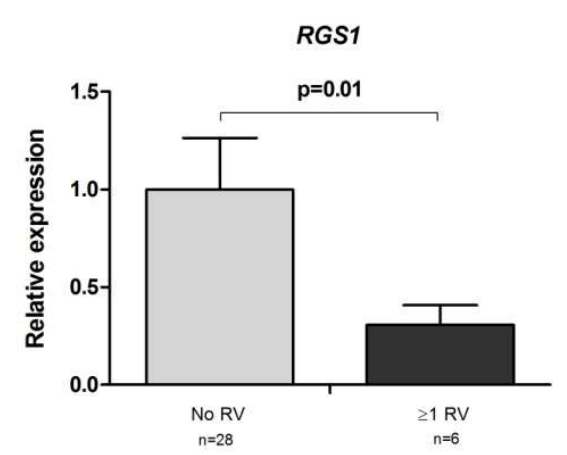

c.

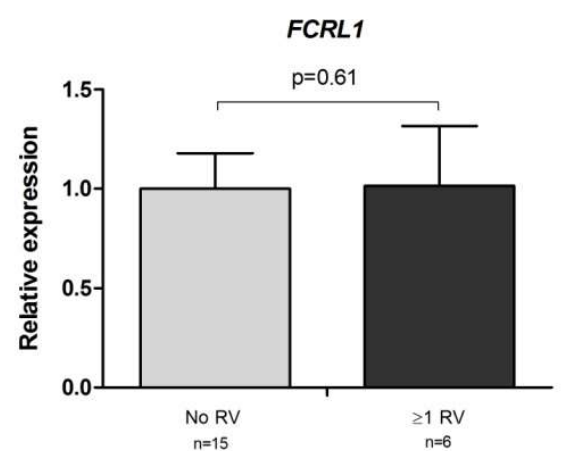

B.

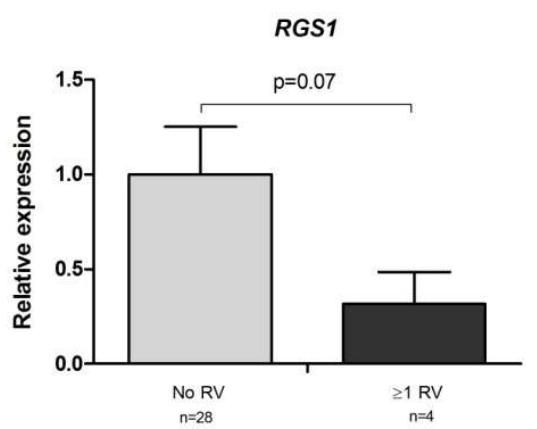

№ RV
Regulatory rare variants

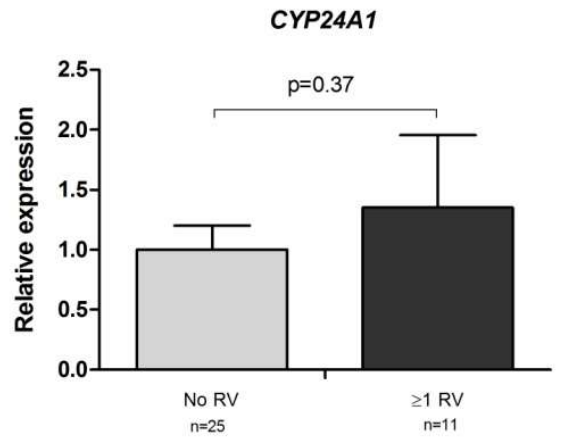

D.
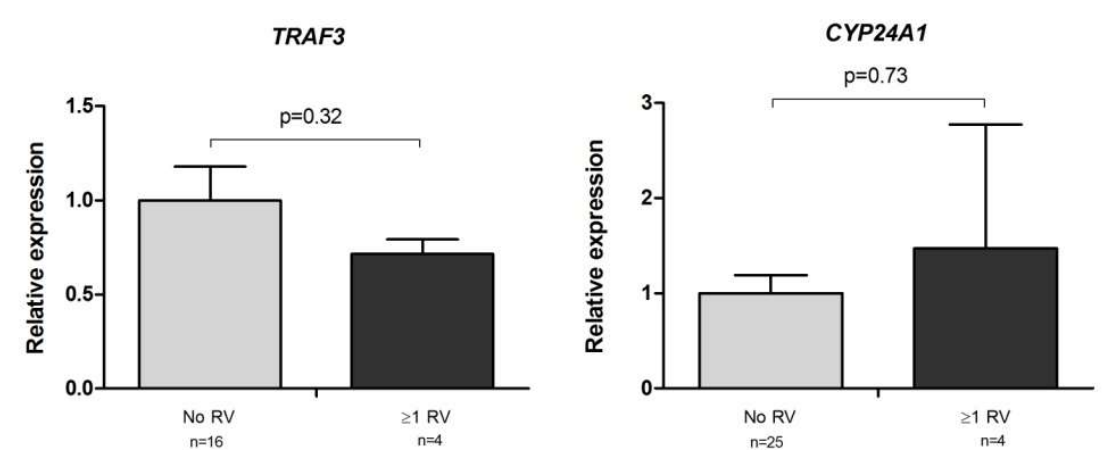
A. All rare variants

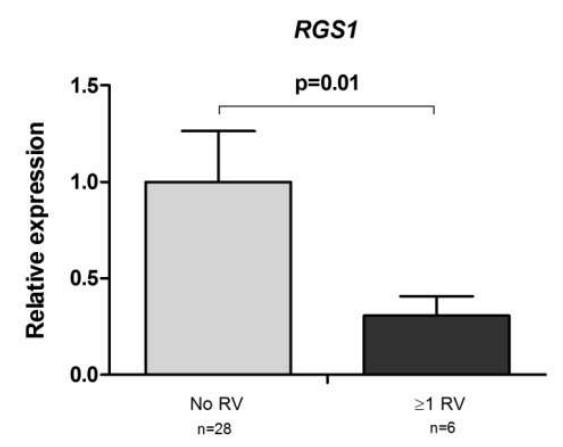

C. Promoter rare variants

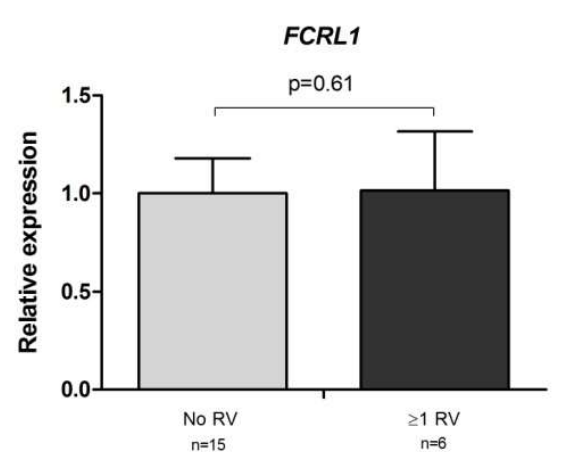

B. Regulatory rare variants
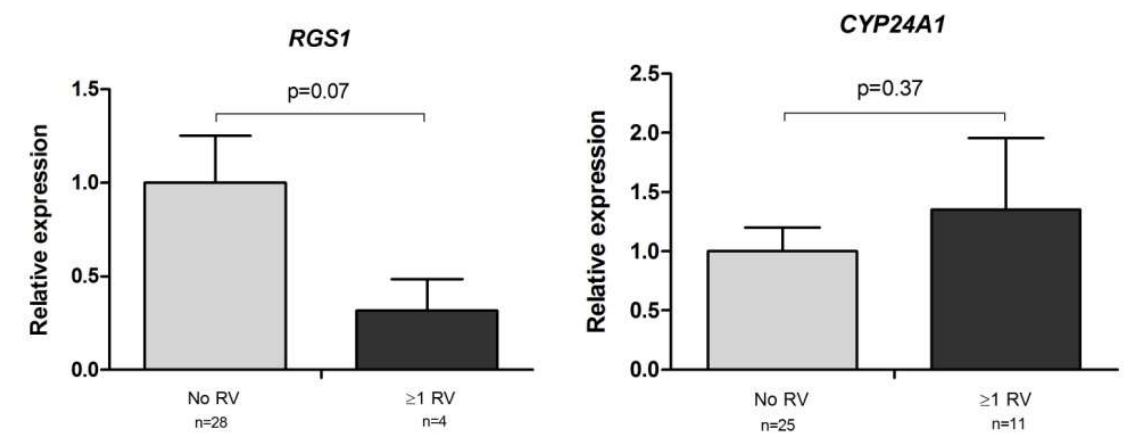

D. UTR rare variants

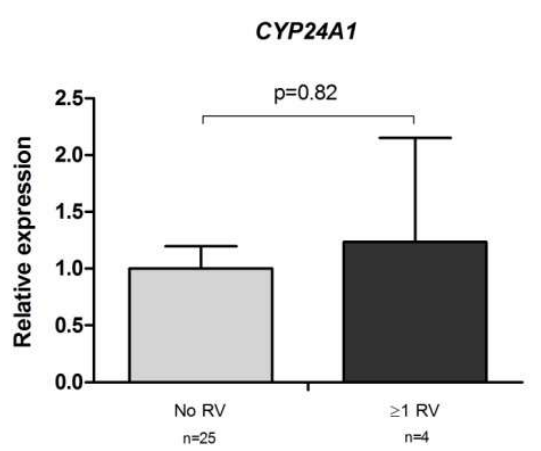

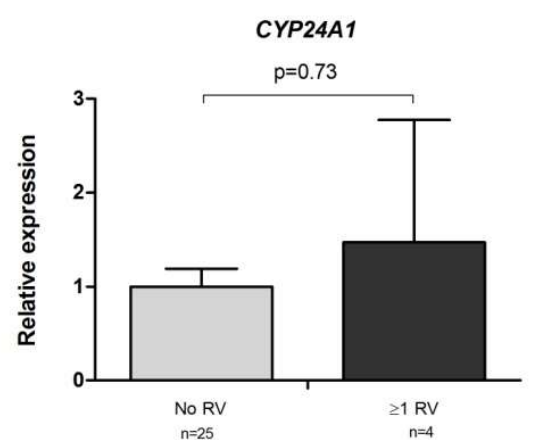


Figure 3

B lymphocytes

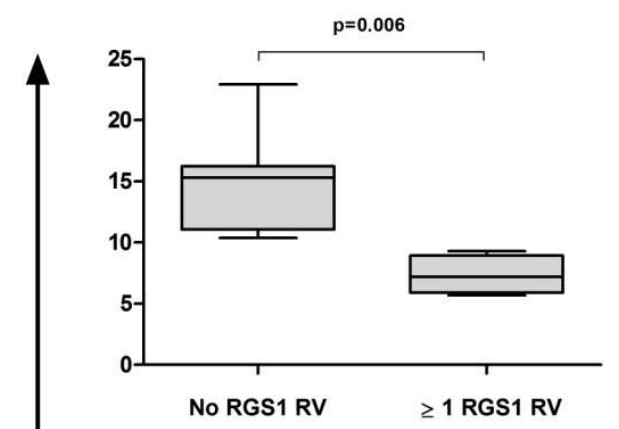

CD4 ${ }^{+}$Iymphocytes

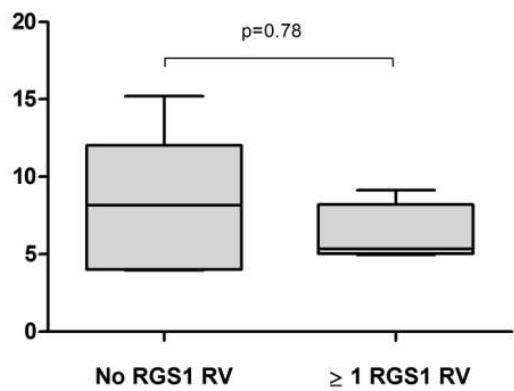

$\underset{0}{+}$

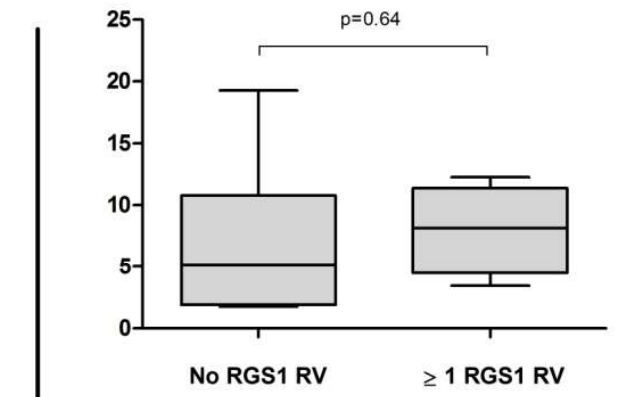

NK cells

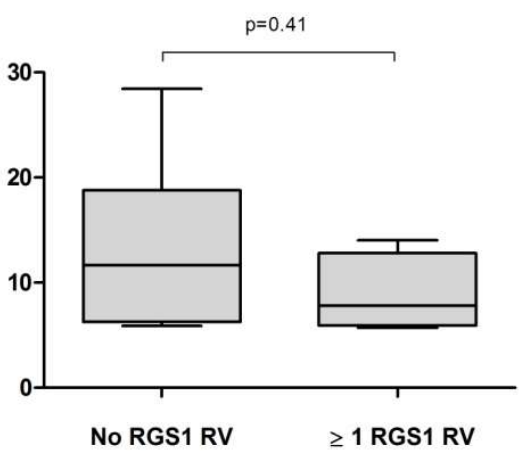

$\mathrm{CD}^{+}$lymphocytes

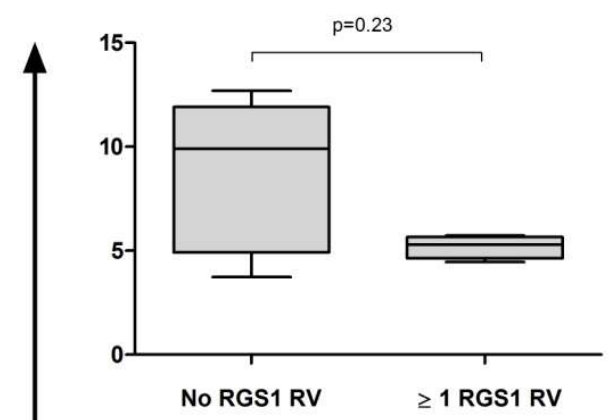

$\mathrm{CD8}^{+}$Iymphocytes

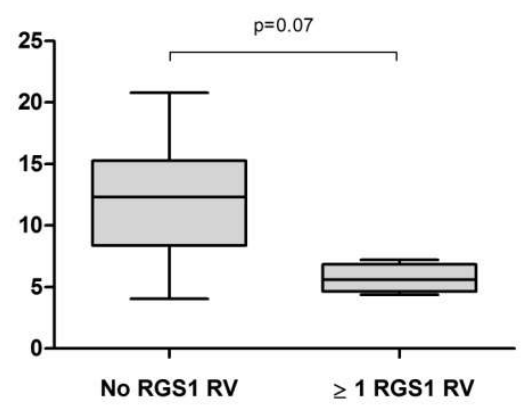

$\underset{0}{+}$

Monocytes

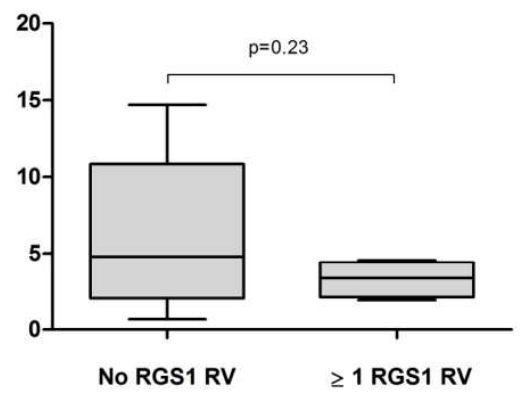

NKT cells

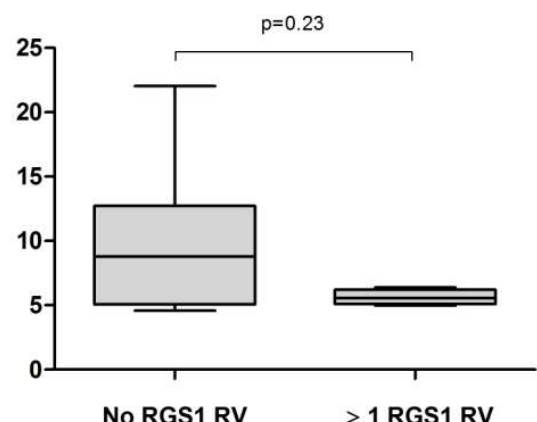


Figure 4

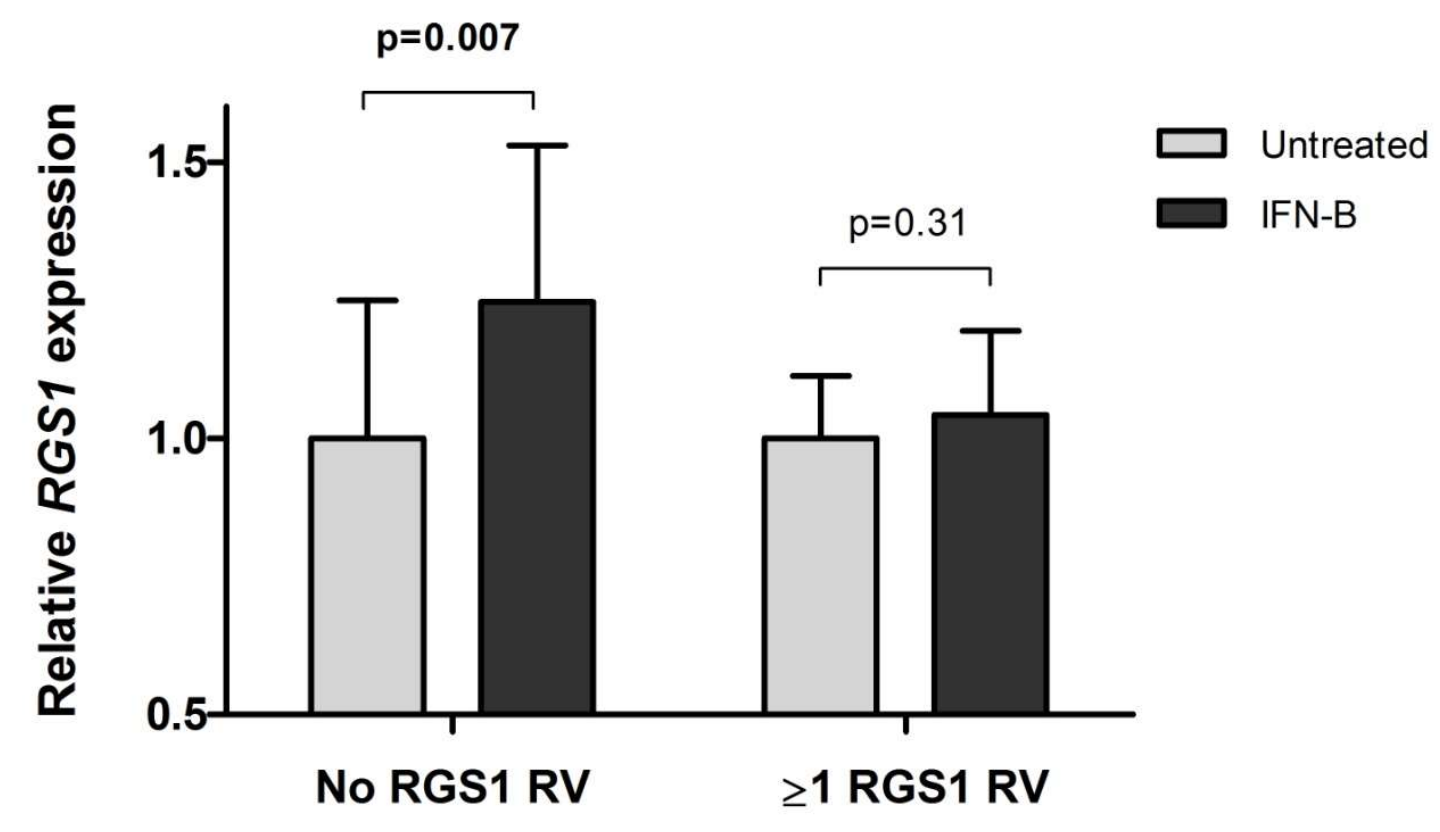


Figure 5

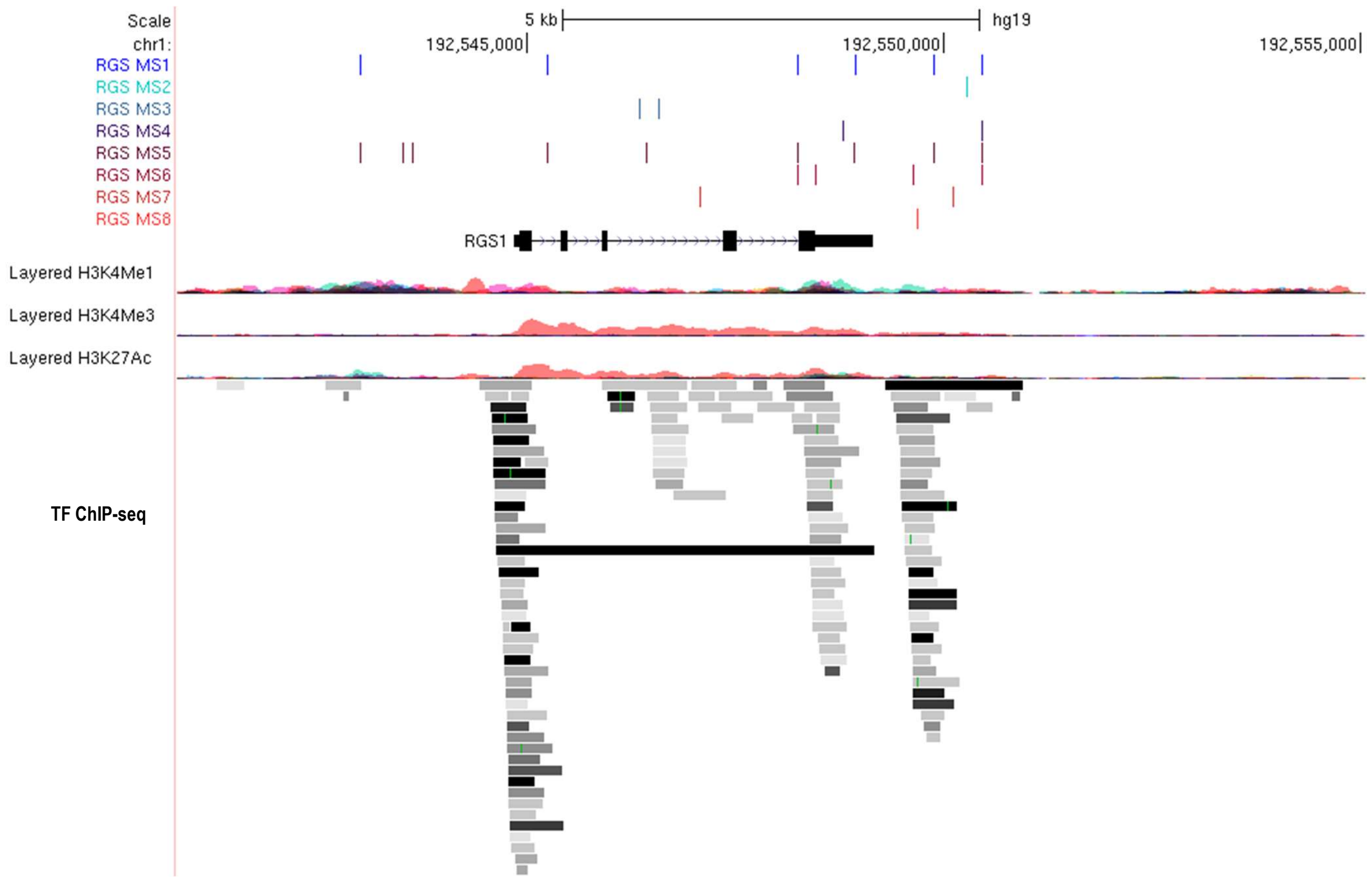

